\title{
Endoplasmic Reticulum Stress in Unfolded Protein Response Signalling
}

\section{Perera JKHM and Dassanayake RS}

Department of Chemistry, University of Colombo, Colombo 03, Sri Lanka

*Corresponding author: Dassanayake RS, Department of Chemistry, University of Colombo, Colombo 03, Sri Lanka, Tel: 94-11-2503 367; E-mail: rsdassanayke@chem.cmb.ac.lk

Rec date: May 12, 2015; Acc Date: May 15, 2015; Pub date: May 19, 2015

Copyright: @ 2015 Perera, et al. This is an open-access article distributed under the terms of the Creative Commons Attribution License, which permits unrestricted use, distribution, and reproduction in any medium, provided the original author and source are credited.

\section{Editorial}

Endoplasmic reticulum (ER) is a central regulator responsible for biosynthesis, folding, quality controlling, maturation and trafficking of proteins $[1,2]$ 'ER stress' is a condition arises when the accumulation capacity of ER for newly synthesized, unfolded proteins is exceeded and activates a highly conserved, intracellular signaling pathway called Unfolded Protein Response (UPR) [2] Multiple signaling pathways like inflammatory and stress signaling systems work in concert with UPR to mitigate ER stress. Prolonged ER may lead to the activation of cell apoptosis pathways. Unresolved ER stress and dysfunctions in UPR have been involved in various metabolic diseases such as obesity, insulin resistance and type 2 diabetes [3] Identifying the molecular mechanisms involved, emerges pathways to design new therapeutics for ER-stress related disorders and resolve metabolic homeostasis [2].

Three ER- located transmembrane proteins: IRE1 (InositolRequiring kinase/ Endoribonuclease protein-1), PERK (Protein kinase RNA- like ER Kinase), and ATF6 (Activating Transcription Factor- 6) are found to be responsible as essential UPR sensors in eukaryotes [2]. In a stress free and well-functioning ER, these proteins are rendered inactive by the binding of GRP78 (Glucose Regulated Proteinof 78 $\mathrm{kDa}$ ) chaperon at their ER lumenal domain. Upon ER stress, GRP78 is released from these UPR sensors rendering them active to bind with unfolded proteins [2].

Depending on the intensity of the ER stress, UPR sensors either activate cell survival pathways or cell apoptotic pathways [3]. Cell survival pathways are activated to mitigate the ER stress by reducing protein synthesis, enhancing protein degradation, increasing chaperon production that facilitate protein folding in the ER lumen [3]. The first pathway is activated by IRE1 which leads to oligomerization, phosphorylation of its kinase domains and splicing of XBP1 (X-boxBinding Protein-1) mRNA to produce an active transcription factor, $\mathrm{XBP} 1 \mathrm{~s}$ which enhances the transcription of UPR target genes associated with protein folding, secretion, quality control and ER associated degradation (ERAD) [1]. PERK activates the second pathway by phosphorylating elf2 $\alpha$ at Ser51 which will attenuate the global protein translation in cells reducing the protein load entering the ER [1]. Moreover phosphorylated elf2 $\alpha$ will give rise to ATF4 which will activate the transcription of UPR genes involved in redox homeostasis, amino acid metabolism and apoptosis induced by ER stress [1]. Translocation of ATF6 into the Golgi apparatus and subsequent cleavage by S1P (serine protease site1 protease) and S2P (metalloprotease site 2 protease) activate the third pathway of UPR and process an active transcription factor, pATF6a (N)4 which induces the expression of ER chaperons and XBP1 [1].

Under prolonged and severe ER stress, IRE1a induces IRE1-ASK1 (Apoptosis Signal regulating Kinase1)-JNK apoptotic pathway which transcriptionally regulate many inflammatory genes whereas PERK- elf2 $\alpha$-ATF4-CHOP pathway is induced by PERK [3]. Recent studies have found that all three UPR sensors can lead to the activation of NFkB-IKK1 inflammatory pathway under these conditions. Under chronic ER stress and activated UPR, toxic accumulation of ROS (Reactive Oxygen Species) can take place due to oxidative stress [1].

ER functions not only in handling proteins but also in charge of controlling synthetic and catabolic pathways of various nutrients such as glucose and lipids. Thus, ER stress and activated UPR signaling have been involved in various metabolic disorders such as obesity, insulin resistance, type 2 diabetes, liver diseases such as NAFLD (Nonalcoholic fatty liver disease) and ALD (Alcoholic fatty liver disease), neurodegenerative diseases, vascular diseases such as Atherosclerosis and Ischemia [2].

Therapeutic approaches are designed as treatments for these disorders by modulating the pathways involved in ER stress and UPR. These approaches emphasize on exploiting chemical chaperons to stabilize misfolded proteins, improve the ER folding capacity and reduce the retention of misfolded proteins. Bortezomib (Velcade) and carfilzomib (Kyprolis) are such two proteasome inhibitor drugs that have been approved by the Food and Drug Administration (FDA) in United States for the treatment of multiple myeloma [2]. These two drugs enhance the development of ER stress in multiple myeloma cells which ultimately leads to cell death. Tumor cells have also been shown to induce ER stress and thus UPR pathway can be devised as a potential target for therapeutics for cancer.3Several efforts have also been undertaken to design drugs which target a specific arm of UPR. For an instance, experiments have been conducted to modulate the RNase activity of IRE1 with the use of several chemicals which also have been suggested as potential treatments for multiple myeloma [4].

Development of genetically engineered model organisms has open up pathways to identify physiological and pathological impacts of UPR on human physiology and metabolic diseases. Explorations conducted using PERK- specific kinase inhibitors [4] in xenograft mouse models have shown an inhibition of tumor growth. Therefore, changes made in approaching the modulation of ER stress can lead to the identification of different treatment patterns for various diseases.

Since UPR signaling provides dual biological functions in both survival and apoptosis, designing specific therapeutics targeting only ER stress, have found to be difficult [3]. Further investigations are conducted to identify novel therapeutic targets which will facilitate cell survival pathways without inducing the apoptotic pathways [3]. Thus, it is important to comprehend how each component in UPR and its cross- talks with other signaling pathways are related to the pathogenesis of metabolic disorders [4]. 
Citation: Perera JKHM, Dassanayake RS (2015) Endoplasmic Reticulum Stress in Unfolded Protein Response Signalling. Biochem Anal Biochem 4: e157. doi:10.4172/2161-1009.1000e157

Page 2 of 2

\section{References}

1. Hotamisligil GS (2010) Endoplasmic reticulum stress and the inflammatory basis of metabolic disease. Cell 140: 900-917.

2. Lee J, Ozcan U (2014) Unfolded protein response signaling and metabolic diseases. J Biol Chem 289: 1203-1211.
3. Kadowaki H, Nishitoh H (2013) Signaling pathways from the endoplasmic reticulum and their roles in disease. Genes (Basel) 4: 306-333.

4. Wang S, Kaufman RJ (2012) The impact of the unfolded protein response on human disease. J Cell Biol 197: 857-867. 